\title{
Annexin A1 is a potential biomarker of bone metastasis in small cell lung cancer
}

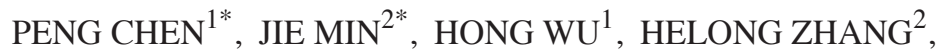 \\ CHAOLI WANG $^{1}$, GUANGGUO TAN ${ }^{1}$ and FENG ZHANG ${ }^{2}$ \\ ${ }^{1}$ Department of Pharmacy, Air Force Military Medical University; ${ }^{2}$ Department of Oncology, Tangdu Hospital, \\ Air Force Military Medical University, Xi'an, Shaanxi 710000, P.R. China
}

Received May 21, 2020; Accepted November 20, 2020

DOI: $10.3892 / \mathrm{ol} .2020 .12402$

\begin{abstract}
Small cell lung cancer (SCLC) is a subtype of lung cancer with a poor prognosis, with bone metastasis being one of the main causes of treatment failure. Therefore, investigating new biomarkers associated with bone metastasis may result in positive treatment outcomes. The present study detected the expression levels of annexin A1 (ANXA1) in the serum of 82 patients with SCLC using ELISA. ANXA1 expression in patients with SCLC with bone metastasis was significantly higher compared with that in patients without bone metastasis. Receiver operating characteristic analysis revealed that ANXA1 expression was significant in the diagnosis of bone metastasis in SCLC. ANXA1 was inhibited in SBC-5 cells and overexpressed in SBC-3 cells. Results revealed that ANXA1 was able to enhance SCLC cell proliferation, invasion, migration and bone adhesion in vitro. In vivo xenograft bone metastasis assays indicated that ANXA1 had the potential to promote the bone-metastasis ability of SCLC cells in NOD/SCID mice. Furthermore, ANXA1 increased parathyroid hormone-related protein secretion and enhanced Smad 2 phosphorylation following TGF- $\beta$ treatment in SCLC cells. Overall, ANXA1 may be involved in the pathogenesis of bone metastasis in SCLC and may be a potential biomarker for the diagnosis of SCLC.
\end{abstract}

\section{Introduction}

Lung cancer is one of the most frequently diagnosed malignant tumors and the leading cause of cancer-associated death worldwide. For example, in the United States lung cancer

Correspondence to: Dr Feng Zhang, Department of Oncology, Tangdu Hospital, Air Force Military Medical University, 569 Xinsi Road, Xi'an, Shaanxi 710000, P.R. China

E-mail: zhangf83@qq.com

${ }^{*}$ Contributed equally

Key words: annexin A1, small cell lung cancer, bone metastases, biomarker, phospholipids-binding proteins caused more deaths than breast, prostate, colorectal and brain cancer combined in 2017, and is projected to account for one-quarter of all cancer-associated deaths in 2020 (1). Histologically, lung cancer can be classified as small cell lung cancer (SCLC) and non-SCLC. SCLC is considered to have the poorest prognosis among all types of lung cancer due to its rapid growth and early distant metastases; clinically, around two-thirds of patients with SCLC present with multiple organ metastases, particularly to the bone (2). Bone metastases are often associated with skeletal-associated events, including pain, hypercalcemia, fracture and nerve compression syndromes, all of which may decrease the quality of life of patients $(3,4)$. The occurrence of bone metastasis directly affects the choice of treatment options (5). Therefore, timely diagnosis of bone metastasis is important to improve the treatment of SCLC. At present, the diagnosis of bone metastasis mainly depends on imaging examination, such as bone scans and computed tomography (CT), which are harmful to patients and not economical; however, due to the rapid progression of SCLC, frequent monitoring of the status of bone metastases is clinically required (6). Therefore, finding biomarkers associated with bone metastasis is important to improve the diagnosis and treatment of patients with SCLC.

Annexin A1 (ANXA1), a member of calcium-dependent phospholipid-binding proteins, serves a role in regulating cell proliferation, apoptosis, phagocytosis and carcinogenesis $(7,8)$. Studies have demonstrated that ANXA1 expression is different in various types of tumor tissue $(8,9)$. For example, ANXA1 expression is upregulated in liver, colorectal and pancreatic cancer, but downregulated in prostate, esophageal and cervical cancer (9). Abnormal ANXA1 expression and the changes of its location may be associated with the differentiation and metastases of malignant tumors $(10,11)$. ANXA1 may be a potential new biomarker for the diagnosis and treatment of tumors; however, the role of ANXA1 in the pathogenesis of SCLC remains unclear. In addition, ANXA1 is involved in regulating bone development and the bone marrow microenvironment $(12,13)$. However, whether this indicates that ANXA1 participates in the bone metastases of tumors requires further confirmation.

The present study analyzed the association between the expression levels of ANXA1 in serum and the clinical characteristics of patients with SCLC, and discussed the diagnostic 
value of ANXA1 expression in bone metastasis. To further confirm the association between ANXA1 and bone metastasis, ANXA1 was overexpressed in SBC-3 cells and inhibited in SBC-5 cells, and the effects of ANXA1 on the biological behavior of SCLC cells and bone metastasis-associated signaling pathways were investigated.

\section{Materials and methods}

Patients and specimens. Serum samples were collected from 82 patients with SCLC who were admitted to Tangdu Hospital of the Air Force Military Medical University (Xi'an, China) between March 2016 and May 2017, and ANXA1 1 expression was detected using a Human Annexin A1 ELISA kit (cat. no. ab222868; Abcam). The present study was based on a cohort of consecutive patients with histopathological diagnosis of SCLC, without inflammatory disease and who had never received chemotherapy or radiotherapy. Detailed information was obtained from the medical records of the enrolled patients in a computerized registry database, including patient age, sex and smoking history. Tumor assessment was performed using $\mathrm{CT}$, radionuclide bone scanning or integrated positron emission tomography-CT. Magnetic resonance imaging or enhanced CT was performed is symptomatic patients. At least one radiologist and one physician confirmed the diagnosis. All tumors were staged according to the pathological tumor/node/metastasis (pTNM) classification (8th edition) of the Union for International Cancer Control (UICC) (14). The study protocol was approved by the ethical review board of Tangdu Hospital. All patients provided written informed consent for use of their medical records and samples for research purposes.

Cell lines and culture. The human SCLC SBC- 3 and SBC-5 cell lines were provided by Professor Saburo Sone and Professor Seiji Yano (University of Tokushima School of Medicine, Tokushima, Japan). Both cell lines were cultured in RPMI-1640 medium supplemented with $10 \%$ heat-inactivated FBS (both Gibco; Thermo Fisher Scientific, Inc.) at $37^{\circ} \mathrm{C}$ in an atmosphere of $5 \% \mathrm{CO}_{2}$.

Transfection. For ANXA1-knockdown in SBC-5 cells, lentiviral vectors for human ANXA1 short hairpin RNAs (shRNAs) were designed according to the human ANXA1 mRNA sequence (GenBank accession no. NM-000700): shANXA1-1, 5'-AACCATCATTGACATTCTA-3'; shANXA1-2, 5'-CTT GTATGAAGCAGGAGAA-3'; shANXA1-3, 5'-AGCGCA ATTTGATGCTGAT-3'; shANXA1-4, 5'-ATTCTATCA GAAGATGTAT-3'; and negative control (non-targeting), 5'-TTCTCCGAACGTGTCACGT-3'. ANXA1 and negative control shRNAs were constructed, packed and purified by Shanghai GeneChem Co., Ltd.. The second generation of self-inactivated lentivirus packaging system was used for virus packaging, which involved three plasmids (Shanghai GeneChem Co., Ltd.): Tool vector plasmid GV248 carrying the target sequence, pHelper 1.0 (carrying gag, pol and rev genes) and pHelper 2.0 (carrying the VSV-G gene). Viral vector generation was obtained by co-transfection of $5 \times 10^{6} 293 \mathrm{~T}$ cells (Shanghai GeneChem Co., Ltd.)/15 ml medium (DMEM with 10\% FBS; Gibco; Thermo Fisher Scientific, Inc.) with $20 \mu \mathrm{g}$ GV248, $15 \mu \mathrm{g}$ pHelper1.0 and $10 \mu \mathrm{g}$ pHelper 2.0 on $10-\mathrm{cm}$ plates. To overexpress ANXA1 in SBC-3 cells, the cDNA of ANXA1 was transfected into the GV166 virus to build the ANXA1/GV166-LV plasmid, which was then packed into lentiviral vectors. SBC-3 cells and SBC-5 cells were seeded in 6-well plates before transfection. When the cells reached $\sim 80 \%$ confluence, SBC- 5 cells were either transfected with ANXA1 shRNAs (SBC-5-shANXA1) or the negative control shRNA (SBC-5-NC), and SBC-3 cells were transfected with pcDNA-ANXA1 (SBC-3-ANXA1) or the negative control (empty) vector by co-incubating with Enhance Infection Solution (Shanghai GeneChem Co., Ltd.) for $12 \mathrm{~h}$ at $37^{\circ} \mathrm{C}$ at a multiplicity of infection of 20, after which the medium was replaced by fresh RPMI-1640 with 10\% FBS. Three days after transfection, cells were selected using $0.5 \mu \mathrm{g} / \mathrm{ml}$ puromycin and harvested for subsequent experiments.

$R T-q P C R$. Total RNA was extracted from cells using TRIzol ${ }^{\circledR}$ reagent (Invitrogen; Thermo Fisher Scientific, Inc.). RT was performed toobtain cDNA using the SuperScriptIIIFirst-Strand Synthesis kit (Invitrogen; Thermo Fisher Scientific, Inc.) according to the manufacturer's protocol. mRNA expression in each cell line was standardized to $\beta$-actin. The following primer pairs were synthesized by Shanghai GeneChem Co., Ltd., for qPCR: ANXA1 forward, 5'-TTTTGGCATCAAGAA CTAAC-3' and reverse, 5'-CCTCAGATCGGTCACCCT-3'; parathyroid hormone-related protein (PTHrP) forward, 5'-GGAGACTGGTTCAGCAGTGG-3' and reverse, 5'-TTG TCATGGAGGAGCTGATG-3'; $\beta$-actin forward, 5'-ATCGTG CGTGACATTAAGGACAAG-3' and reverse, 5'-AGGAAG GAAGGGCTGGAAGAGTG-3'. qPCR was preformed using SYBR Premix Ex Taq ${ }^{\mathrm{TM}}$ II (Takara Bio, Inc.). The following thermocycling conditions were used for qPCR: Initial denaturation at $95^{\circ} \mathrm{C}$ for $3 \mathrm{~min} ; 35$ cycles of $95^{\circ} \mathrm{C}$ for $15 \mathrm{sec}, 60^{\circ} \mathrm{C}$ for $30 \mathrm{sec}$ and $72^{\circ} \mathrm{C}$ for $1 \mathrm{~min}$; extension at $72^{\circ} \mathrm{C}$ for $7 \mathrm{~min}$. Each sample was detected in triplicate, and a melting curve was analyzed to confirm amplification specificity. Relative mRNA expression was calculated using the $2^{-\Delta \Delta C q}$ method (15).

Western blotting. Harvested cells were washed once with PBS and lysed to extract total cellular protein using RIPA buffer (Beyotime Institute of Biotechnology). The proteins were boiled for $5 \mathrm{~min}$ and protein concentration was quantified using a Micro-BCA protein assay. Subsequently, $20 \mu \mathrm{g} / \mathrm{lane}$ protein was subjected to $10 \%$ SDS-PAGE and transferred to PVDF membranes. The membranes were then blocked in $5 \%$ skimmed milk for $1 \mathrm{~h}$ at room temperature and incubated overnight at $4^{\circ} \mathrm{C}$ with the following primary antibodies: Anti-ANXA1 (1:500; cat. no. AMAB90558; Sigma-Aldrich; Merck KGaA), anti-PTHrP (1:200; cat. no. sc-53936; Santa Cruz Biotechnology, Inc.), anti-Smad2 (1:500, cat. no. 5339; Cell Signaling Technology, Inc.), anti-phosphorylated (p)-Smad2 (1:500; cat. no. 18338; Cell Signaling Technology, Inc.) and anti- $\beta$-actin (1:1,000; cat. no. A1978; Sigma-Aldrich; Merck KGaA). Subsequently, the membranes were incubated with peroxidase-conjugated goat anti-rabbit IgG (1:5,000; cat. no. ZB-2301; OriGene Technologies, Inc.) or goat anti-mouse IgG (1:5,000; cat. no. ZB-2305; OriGene Technologies, Inc.) secondary antibodies for $1 \mathrm{~h}$ at room temperature. Finally, the target proteins were visualized by chemiluminescence (Pierce; Thermo Fisher Scientific, Inc.) 
and measured using ImageJ software (version 1.52a; National Institutes of Health).

MTT assay. SBC-3 and SBC-5 cells in the logarithmic phase were harvested and seeded into 96 -well plates $\left(5 \times 10^{3}\right.$ cells/well). To assess the relative cell number, $20 \mu \mathrm{l}$ of $5 \mathrm{mg} / \mathrm{ml} \mathrm{MTT} \mathrm{solu-}$ tion was added to the wells and cells were incubated for $3 \mathrm{~h}$ at $37^{\circ} \mathrm{C}$. The supernatant was removed and $150 \mu \mathrm{l}$ DMSO was added to each well to dissolve the blue formazan crystals. The absorbance was determined at a wavelength of $490 \mathrm{~nm}$ using a Model 680 microplate reader (Bio-Rad Laboratories, Inc.). To draw the growth curve, the cell number was measured at 24, 48, 72, 96 and 120 h. For accuracy, each assay contained six replicates and was repeated three times.

Colony formation assay. Single-cell suspensions were plated in a $35-\mathrm{mm}$ diameter plate at a density of $1 \times 10^{3}$ cells/plate and incubated at $37^{\circ} \mathrm{C}$ with $5 \% \mathrm{CO}_{2}$ for 7 days. Subsequently, at room temperature, the colonies were fixed with $4 \%$ formaldehyde for $15 \mathrm{~min}$, stained with $0.5 \%$ crystal violet for $15 \mathrm{~min}$ and counted manually using a light microscope (one colony contained $\geq 50$ cells). Three independent plates were set up for accuracy.

Cell cycle assay. The cells were resuspended at $1 \times 10^{6}$ cells $/ \mathrm{ml}$, fixed and permeabilized with $75 \%$ ethanol at $4^{\circ} \mathrm{C}$ overnight and washed with cool PBS. Subsequently, the cells were stained with staining solution $(50 \mu \mathrm{g} / \mathrm{ml}$ propidium iodide and $20 \mu \mathrm{g} / \mathrm{ml}$ RNase in PBS; Sigma-Aldrich; Merck KGaA) at $37^{\circ} \mathrm{C}$ for $30 \mathrm{~min}$ in the dark. The analysis of cellular DNA content was performed using a flow cytometer (FACSCalibur; BD Biosciences) at an excitation wavelength of $488 \mathrm{~nm}$. The distribution of these cells was analyzed using CellQuest v3.3 and ModFit v3.1 softwares (BD Biosciences).

In vitro migration and invasion assay. Both the migratory and invasive abilities of cells were assessed using Transwell inserts $(8-\mu \mathrm{m}$ pore size) in a 24 -well plate (Corning, Inc.). The cells were starved for $24 \mathrm{~h}$ prior to the migration assay

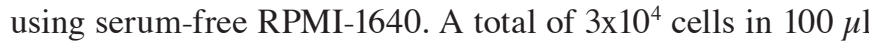
RPMI-1640 with 0.1\% BSA (Sigma-Aldrich; Merck KGaA) were seeded in triplicates into the upper chamber of the Transwell insert. RPMI-1640 (500 $\mu \mathrm{l})$ with 10\% FBS was added into the lower chamber as a chemoattractant. Following $48 \mathrm{~h}$ of incubation at $37^{\circ} \mathrm{C}$, cells that did not migrate through the membranes were gently removed using a cotton swab. At room temperature, cells adhering to the lower surface of the membranes were fixed with $4 \%$ formaldehyde for $15 \mathrm{~min}$ and stained with $0.5 \%$ crystal violet for $10 \mathrm{~min}$. Finally, migrated cells were imaged at x400 magnification using a light microscope and counted manually in five randomly selected fields per well. For the invasion assay, the inserts were coated with $70 \mu 1$ Matrigel ${ }^{\circledR}$ (1:8 dilution with serum-free RPMI-1640; BD Biosciences) at $37^{\circ} \mathrm{C}$ for $30 \mathrm{~min}$ before the cells were seeded into the upper chamber. Subsequent steps were performed as aforementioned for the migration assay.

In vitro bone adhesion ability assay. A total of 30 female NOD-SCID mice (age, 5 days old; weight, 4.4-5.8 g) were obtained from the Laboratory Animal Research Center of the Air Force Military Medical University (Xi'an, China) and sacrificed by inhaling carbon dioxide $\left(\mathrm{CO}_{2}\right.$ flow rate, $20 \%$ of the container volume per min). As previously described (16), skulls of mice were dissected, swabbed with alcohol wipes and soaked in PBS containing $1 \times 10^{4} \mathrm{U} / \mathrm{ml}$ penicillin for $24 \mathrm{~h}$ at room temperature. Once the supernatant was removed following centrifugation at $1,000 \mathrm{x}$ for $15 \mathrm{~min}$ at $20^{\circ} \mathrm{C}$, collagenase I (5 mg/ml; $400 \mu \mathrm{l}$; Sigma-Aldrich; Merck KGaA), collagenase II (5 mg/ml; $400 \mu \mathrm{l}$; Sigma-Aldrich; Merck KGaA) and $0.05 \%$ trypsin $(200 \mu \mathrm{l})$ were added to digest the skulls at $37^{\circ} \mathrm{C}$ for $2 \mathrm{~h}$. The skulls were then washed with PBS twice, and were cut into pieces of $3 \times 3 \mathrm{~mm}^{2}$ for subsequent experimentation. Agar (1\%; $0.5 \mathrm{ml} /$ well) was added into a 24 -well plate, and the bone pieces were fixed to the solidified agar surface. Subsequently, each well was washed with RPMI-1640 without FBS and incubated in $1 \mathrm{ml}$ RPMI-1640 with $10 \%$ FBS in an incubator for $30 \mathrm{~min}$ at $37^{\circ} \mathrm{C}$. A total of $1 \times 10^{4}$ cells were planted onto the skulls and incubated for $24 \mathrm{~h}$ at $37^{\circ} \mathrm{C}$. Subsequently, the bone pieces were taken out, washed with PBS three times, fixed with $95 \%$ alcohol for 10 min and stained with $0.5 \%$ crystal violet for $15 \mathrm{~min}$, all at room temperature. Finally, cells adhering to the bone surface were visualized under a light microscope (magnification, x100). Cells adhering to the bone were digested using trypsin and resuspended in PBS to measure the optical density at a wavelength of $600 \mathrm{~nm}$ using a spectrophotometer.

In vivo xenograft bone metastasis assay. A total of 10 female NOD/SCID mice (age, 3-5 weeks old; weight, 19-24 g) were obtained from the Laboratory Animal Center of the Air Force Military Medical University and raised under germ-free conditions (temperature, $22^{\circ} \mathrm{C}$; ventilation rate, $15 / \mathrm{h}$; light/dark cycle, $12 / 12 \mathrm{~h}$; food was sterilized with Cobalt-60 irradiation and water was autoclaved; access to food and water was ad libitum). SBC-5-NC/SBC-5-shANXA1 cells were harvested from $80-90 \%$ confluent conditions, washed twice and resuspended in PBS. Subsequently, $2 \times 10^{6}$ cells in $200 \mu \mathrm{l}$ PBS were implanted into the NOD/SCID mice (4-6 weeks old) via intravenous injection. The mice were divided into two groups according to the expression levels of ANXA1. On the 35th day post-inoculation, mice were anesthetized by intraperitoneal injection of $1 \%$ pentobarbital (40 mg/kg). Resultant bone metastases were visualized using X-ray irradiation. At the end of the experiment, the mice were sacrificed by inhaling carbon dioxide $\left(\mathrm{CO}_{2}\right.$ flow rate, $20 \%$ of the container volume per min). All animal experimental procedures, including the aforementioned bone adhesion ability assays, were approved by the Animal Ethics Committee of the Air Force Military Medical University and were in accordance with the 'Animal Research: Reporting In Vivo Experiments' guidelines (17).

ELISA and TGF- $\beta$ treatment. The cells at $80 \%$ confluence were harvested, plated into 6 -well tissue culture plates $\left(1 \times 10^{5}\right.$ cells $/ 2 \mathrm{ml} /$ well $)$ and incubated for $24 \mathrm{~h}$ at $37^{\circ} \mathrm{C}$ with $5 \% \mathrm{CO}_{2}$. Culture supernatants were collected and the concentrations of PTHrP were determined using the PTHrP ELISA kit (cat. no. CSB-E08649h; Cusabio Technology LLC). For experiments involving TGF- $\beta$ treatment, $0.5 \mathrm{ng} / \mathrm{ml}$ recombinant human TGF- $\beta 1$ (cat. no. rcyc-htgfb1; InvivoGen) was 
added to the medium and incubated for $12 \mathrm{~h}$ at $37^{\circ} \mathrm{C}$ with $5 \% \mathrm{CO}_{2}$.

Statistical analysis. The data are presented as the mean \pm standard deviation. The Kruskal-Wallis test followed by Bonferroni correction for multiple comparisons was used to compare ANXA1 expression in patients with SCLC. Fisher's exact test was used for the association between ANXA1 expression and clinicopathological characteristics. When the variables were normally distributed, unpaired Student's t-test was used to compare two groups and one-way ANOVA was used to compare three groups, followed by Tukey's post hoc test. Receiver operating characteristic (ROC) curve analysis was used to evaluate the diagnostic value of ANXA1 for bone metastasis in SCLC. Data analysis was performed using SPSS software (version 25.0; IBM Corp.). $\mathrm{P}<0.05$ was considered to indicate a statistically significant difference.

\section{Results}

ANXA1 expression is associated with bone metastases in patients with SCLC. Initially, ANXA1 expression was determined in the serum of 82 patients with SCLC (28 females and 54 males; median age, 64 years; age range, 33-83 years). UICC staging evaluation demonstrated stage I disease in 23 patients, stage II disease in 13 patients, stage III disease in 15 patients and stage IV disease in 31 patients. Among the 82 patients with SCLC, distant organ metastasis occurred in 31 cases at initial diagnosis, including 6 cases of brain metastasis, 17 cases of bone metastasis, 13 cases of liver metastasis and 5 cases of adrenal metastasis. ELISA results revealed that ANXA1 expression in the serum ranged between 0.6-6.6 $\mu \mathrm{g} / 1$ (Fig. 1A; median, $2.45 \mu \mathrm{g} / \mathrm{l}$; mean, $2.74 \mu \mathrm{g} / \mathrm{l})$.

Subsequently, the associations between ANXA1 expression and clinicopathological characteristics of the patients were analyzed (Table I). The results revealed that ANXA1 expression was significantly associated with lymphatic invasion $(\mathrm{P}<0.01)$, bone metastasis $(\mathrm{P}=0.03)$ and $\mathrm{TNM}$ stage $(\mathrm{P}<0.01)$. No significant association was observed between ANXA1 expression and age $(\mathrm{P}=0.62)$, sex $(\mathrm{P}=0.82)$, smoking history $(\mathrm{P}=0.48)$, tumor invasion $(\mathrm{P}=0.44)$, metastasis $(\mathrm{P}=0.07)$, liver metastasis $(\mathrm{P}>0.99)$ and brain metastasis $(\mathrm{P}=0.68)$.

To further clarify the specific association between ANXA1 expression and bone metastasis, patients with SCLC were divided into three groups: 'No metastasis' group ( $n=51)$; 'bone metastasis' group $(n=17)$ and 'organ metastasis except bone metastasis' group $(n=14)$. ANXA1 expression in patients with SCLC with bone metastasis was significantly higher compared with the 'no metastasis' and 'organ metastasis except bone metastasis' groups $(3.70 \pm 1.44$ vs. $2.47 \pm 1.56$ and $2.56 \pm 0.89$, respectively; $\mathrm{P}<0.05)$, but there was no significant difference between the latter two groups (Fig. 1A). Furthermore, the results of the ROC curve analysis revealed that ANXA1 expression was of diagnostic value for SCLC bone metastasis (area under the curve, 0.74; 95\% CI, 0.63-0.83; $\mathrm{P}<0.05$ ). When the cut-off value was 2.80 , the sensitivity was $76.47 \%$ and the specificity was $66.15 \%$ (Fig. 1B).

SBC-3 and SBC-5 cell lines have similar genetic backgrounds; however, $\mathrm{SBC}-5$ cells are more prone to bone metastasis (18). Total RNA and protein of SBC-3 and SBC-5
Table I. Clinicopathological associations of ANXA1 expression in 82 patients with small cell lung cancer.

\begin{tabular}{|c|c|c|c|c|}
\hline \multirow[b]{2}{*}{ Category } & \multirow[b]{2}{*}{$\mathrm{N}$} & \multicolumn{2}{|c|}{$\begin{array}{c}\text { ANXA1 } \\
\text { expression }{ }^{\mathrm{a}}\end{array}$} & \multirow[b]{2}{*}{ P-value } \\
\hline & & High & Low & \\
\hline \multicolumn{5}{|c|}{ Age, years } \\
\hline$<60$ & 23 & 13 & 10 & 0.62 \\
\hline$\geq 60$ & 59 & 28 & 31 & \\
\hline \multicolumn{5}{|l|}{ Sex } \\
\hline Male & 54 & 26 & 28 & 0.82 \\
\hline Female & 28 & 15 & 13 & \\
\hline \multicolumn{5}{|c|}{ Smoking history } \\
\hline Yes & 73 & 35 & 38 & 0.48 \\
\hline No & 9 & 6 & 3 & \\
\hline \multicolumn{5}{|c|}{ Tumor invasion } \\
\hline $\mathrm{T} 1-2$ & 62 & 29 & 33 & 0.44 \\
\hline T3-4 & 20 & 12 & 8 & \\
\hline \multicolumn{5}{|c|}{ Lymphatic invasion } \\
\hline N0-1 & 40 & 13 & 27 & $<0.01$ \\
\hline $\mathrm{N} 2-3$ & 42 & 28 & 14 & \\
\hline \multicolumn{5}{|c|}{ Metastasis } \\
\hline Yes & 31 & 20 & 11 & 0.07 \\
\hline No & 51 & 21 & 30 & \\
\hline \multicolumn{5}{|c|}{ Bone metastasis } \\
\hline Yes & 17 & 13 & 4 & 0.03 \\
\hline No & 65 & 28 & 37 & \\
\hline \multicolumn{5}{|c|}{ Liver metastasis } \\
\hline Yes & 13 & 6 & 7 & $>0.99$ \\
\hline No & 69 & 35 & 34 & \\
\hline \multicolumn{5}{|c|}{ Brain metastasis } \\
\hline Yes & 6 & 2 & 4 & 0.68 \\
\hline No & 76 & 39 & 37 & \\
\hline \multicolumn{5}{|c|}{ TNM stage } \\
\hline I-II & 36 & 11 & 25 & $<0.01$ \\
\hline III-IV & 46 & 30 & 16 & \\
\hline
\end{tabular}

aLow and high ANXA1 expression was determined according to the median value. ANXA1, annexin A1.

cells were extracted to examine ANXA1 mRNA and protein expression using RT-qPCR (Fig. 1C) and western blotting (Fig. 1D), respectively. As expected, the mRNA and protein expression levels of ANXA1 in SBC-5 cells were significantly higher compared with those in SBC-3 cells (Fig. 1C and D).

ANXA1 overexpression enhances the proliferation, migration and invasion of SCLC cell lines. RT-qPCR and western blotting revealed that shRNA-2 was the most efficient shRNA, and this was therefore used for subsequent experiments (Fig. S1A and C). ANXA1 cDNA was transfected to overexpress ANXA1 in SBC-3 cells (Fig. S1B and D). The effects of 
A

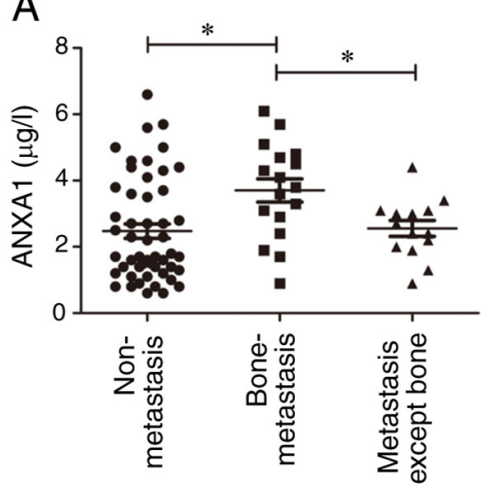

B

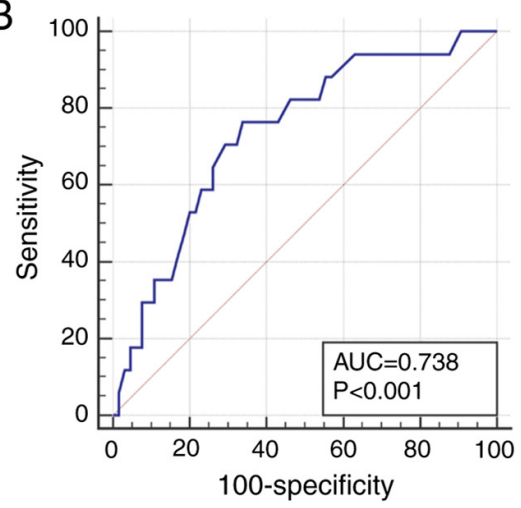

C

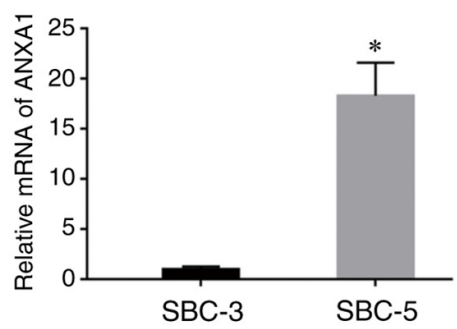

D
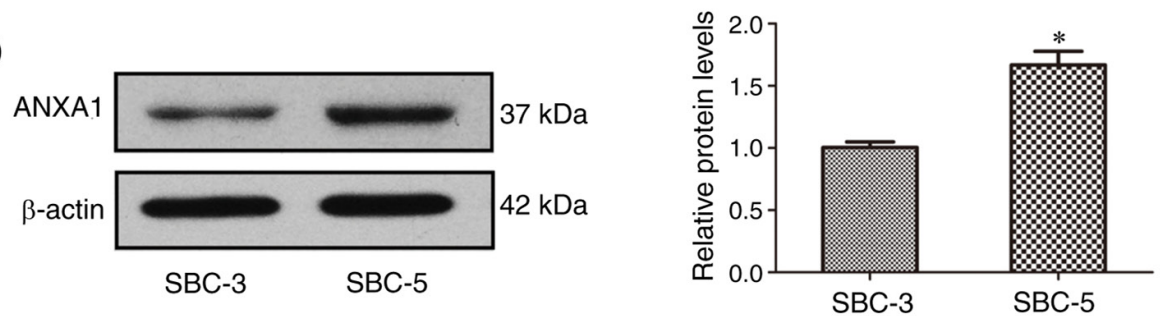

Figure 1. ANXA1 expression in patients with SCLC and cell lines. (A) ANXA1 expression in the serum of patients with SCLC analyzed via ELISA. (B) Receiver operating characteristic curve of ANXA1 expression in the screening of SCLC bone metastasis. (C) Reverse transcription-quantitative PCR and (D) western blot analysis of ANXA1 expression in SBC-3 and SBC-5 cell lines. ${ }^{*} \mathrm{P}<0.05$. BM, bone metastasis; ANXA1, annexin A1; SCLC, small cell lung cancer; AUC, area under the curve.

ANXA1 on cell proliferation were detected using MTT and colony formation assays. In the MTT assay, SBC-3-ANXA1 cells grew at a faster rate compared with SBC-3-vector or parental SBC-3 cells (Fig. 2A). On the other hand, the proliferative rate of SBC-5-shANXA1 cells was lower compared with SBC-5-NC and SBC-5 cells, with a significant difference in the cell proliferation curve observed from day 4 (Fig. 2A). In the planar cloning experiment, colony formation was counted at 1 week after inoculation. ANXA1 overexpression significantly increased cell colony formation ability, while inhibition of ANXA1 expression significantly decreased cell colony formation ability (Fig. 2B).

Cell cycle analysis was further applied to assess the change in cell proliferation following overexpression or inhibition of ANXA1. As shown in Fig. 2C, the percentage of cells in the $\mathrm{G}_{1}$ phase was higher in SBC-3 and SBC-3-vector cells compared with in SBC-3-ANXA1 cells $(61.83 \pm 3.06$ and $61.32 \pm 3.37$ vs. $40.47 .29 \pm 2.44$, respectively; $\mathrm{P}<0.05$ ), while the percentages of cells in $\mathrm{S}$ phase were $23.14 \pm 2.21,24.55 \pm 2.97$ and $38.04 \pm 2.88$ $(\mathrm{P}<0.05)$, respectively. Additionally, SBC-5-shANXA1 cells had a higher proportion of cells in the $\mathrm{G}_{1}$ phase compared with SBC-5 and SBC-5-NC cells (67.59 \pm 2.38 vs. $43.09 \pm 4.25$ and $42.82 \pm 2.33$, respectively; $\mathrm{P}<0.05)$ and a lower proportion in $S$ phase $(22.95 \pm 2.76$ vs. $41.42 \pm 2.05$ and $41.27 \pm 3.71$, respectively; $\mathrm{P}<0.05$ ) (Fig. 2C).

Transwell assays were performed to determine the effects of ANXA1 on the invasion and migration of SCLC cells. ANXA1-transfected SBC-3 cells exhibited significantly increased migratory and invasive abilities compared with controls (Figs. 2D and S2). Consistently, functional inhibition of ANXA1 significantly impaired the migration and invasion of SBC-5 cells (Figs. 2D and S2).
ANXA1 facilitates the adhesion of SCLC cells to bone in vitro and bone metastases in NOD/SCID mice. The effects of ANXA1 on the bone adhesion ability of SCLC cells were detected via a bone adhesion model in vitro. In the attachment assay, the number of SBC-3-ANXA1 cells that adhered to bone was significantly higher compared with controls (Fig. 3A). On the other hand, functional inhibition of ANXA1 significantly impaired the adhesive ability of SBC-5 cells compared with that of controls (Fig. 3B).

Furthermore, an in vivo xenograft bone metastasis model in NOD/SCID mice was established. Five weeks after SBC-5-NC and SBC-5-shANXA1 cells were injected into the tail vein, $\mathrm{X}$-ray images displayed significantly decreased lesions and damage on bones in the ANXA1-inhibition group (Fig. 3C). The bone metastasis ability of cells with high or low ANXA1 expression was quantified by the number of bone metastases $(3.00 \pm 2.51$ vs. $0.63 \pm 1.06$, respectively; $\mathrm{P}<0.05)$. Lung and liver metastases were also observed in mice (data not shown). These results indicated that inhibition of ANXA1 significantly decreased bone metastasis in animal models.

Synthesis and secretion of PTHrP in SCLC cells is mediated by ANXA1. To confirm the association between ANXA1 and bone metastasis at the mechanistic level, the effects of ANXA1 on PTHrP expression, which serves an important role in bone metastasis of SCLC $(19,20)$, were investigated. To elucidate whether modulation of ANXA1 affected PTHrP synthesis, western blotting, RT-qPCR and ELISA were applied to quantify PTHrP levels secreted by SCLC cells. SBC-3-ANXA1 cells secreted significantly higher PTHrP levels compared with parental and control SBC-3 cells, while PTHrP synthesis in 
A
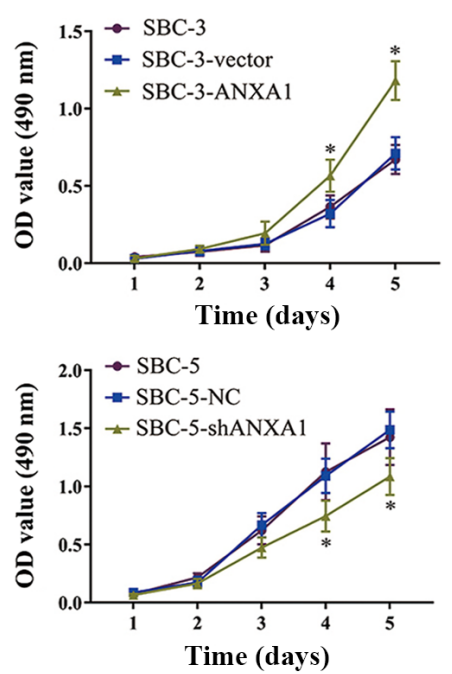

C
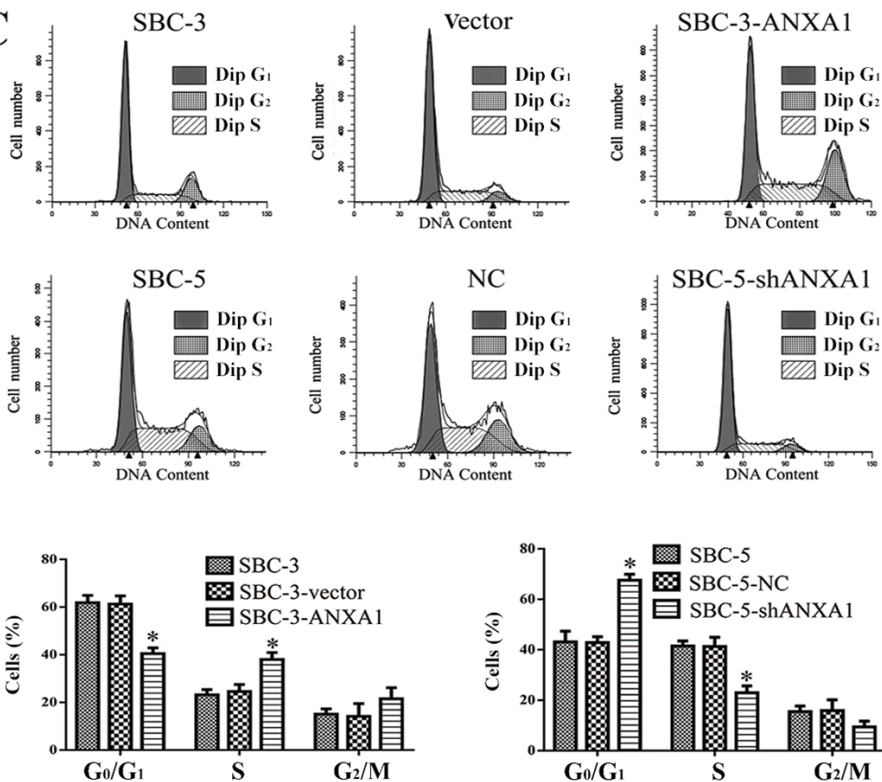

B

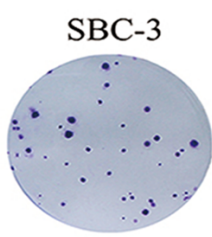

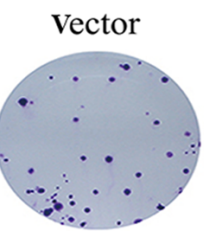
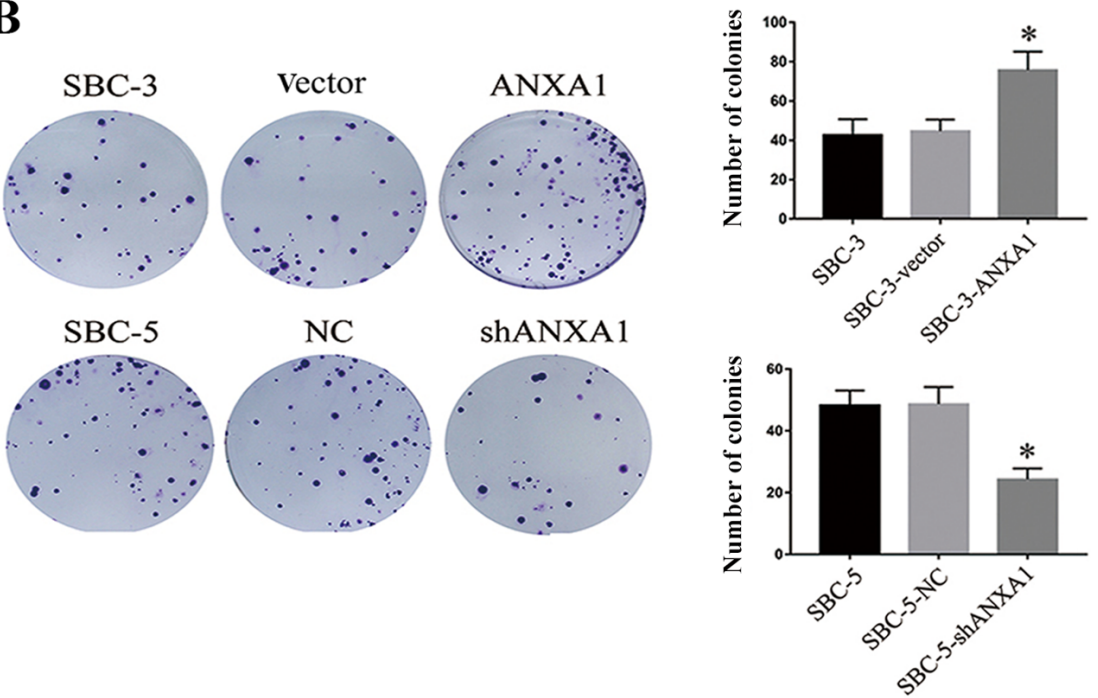

D
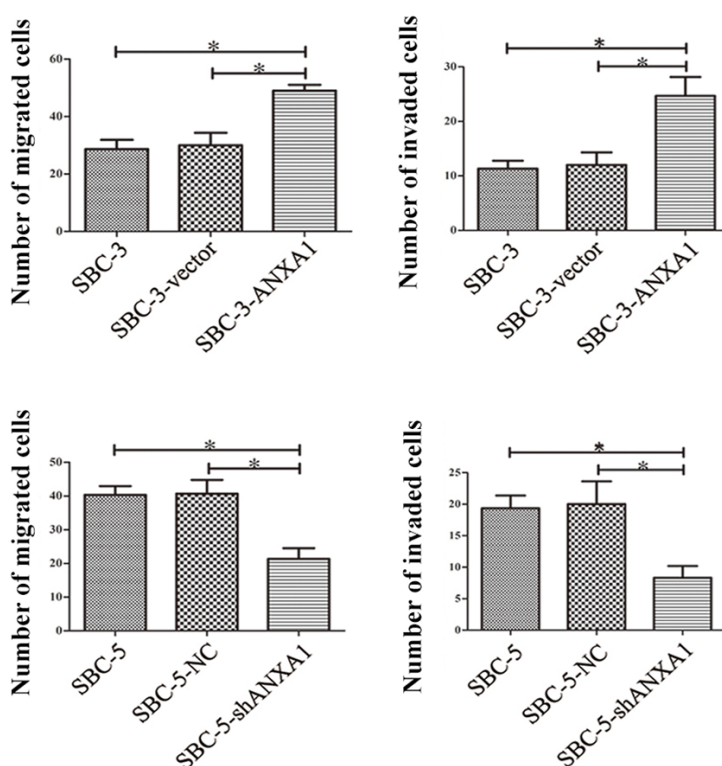

Figure 2. Effects of ANXA1 overexpression or knockdown on SCLC cell proliferation, migration and invasion. Effects of ANXA1 on SCLC cell proliferation detected via (A) MTT and (B) colony formation assays. (C) Effects of ANXA1 on the cell cycle detected via flow cytometry. (D) Effects of ANXA1 on invasion and migration of SCLC cells. ${ }^{*} \mathrm{P}<0.05$ vs. parental or control cells. ANXA1, annexin A1; SCLC, small cell lung cancer; OD, optical density; NC, negative control; sh, short hairpin.

SBC-5-shANXA1 cells was significantly decreased compared with parental and control SBC-5 cells (Fig. 4A-C).

A previous study has demonstrated that $\mathrm{TGF}-\beta / \mathrm{Smad}$ signaling participates in regulating PTHrP secretion and serves an important role in bone metastasis (21). Therefore, the expression levels of Smad 2 and p-Smad 2 after exposure to TGF- $\beta$ were detected. As shown in Fig. 4D, TGF- $\beta$ treatment significantly increased Smad2 phosphorylation in the cells. ANXA1 transfection into SBC-3 cells enhanced the function of TGF- $\beta$ to induce Smad 2 phosphorylation, while ANXA1-knockdown in SBC-5 cells impaired Smad2 phosphorylation in response to TGF- $\beta$ exposure.

\section{Discussion}

Bone metastasis hinders the treatment of SCLC, and timely diagnosis of bone metastasis is a prerequisite to improve treatment effects. Therefore, it is of scientific importance and clinical value to identify biomarkers associated with bone metastasis in SCLC.

Compared with other subtypes of lung cancer, SCLC treatment mainly depends on radiotherapy and chemotherapy; hence, access to tumor tissue samples is limited (22). By contrast, blood samples are easy to obtain, and several biomarkers in the blood, such as lactate dehydrogenase, can be effective in reflecting bone metastases (23). Therefore, serum samples of patients with SCLC were used in the present study to study biomarkers for bone metastasis. By detecting the expression levels of ANXA1 in the serum of 82 patients with SCLC, a strong association was identified between ANXA1 expression and bone metastasis. First, compared with patients with SCLC without metastasis, ANXA1 expression was significantly increased in patients with bone metastasis, while no significant increase in ANXA1 expression was 
A

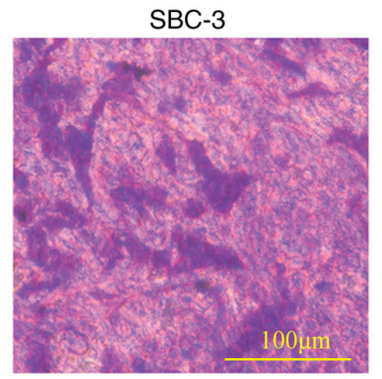

$\mathrm{B}$

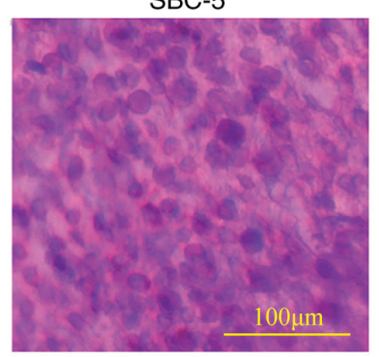

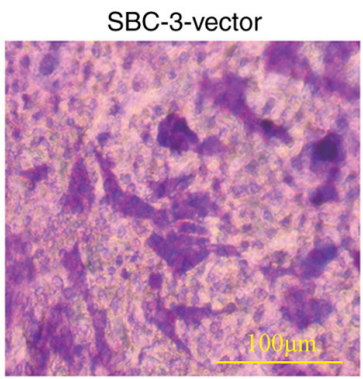

SBC-5-NC

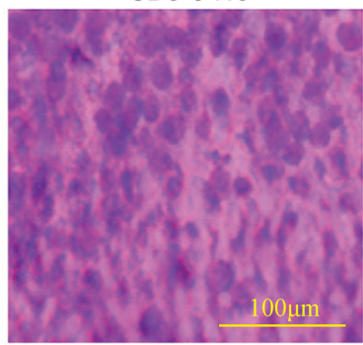

SBC-3-ANXA1

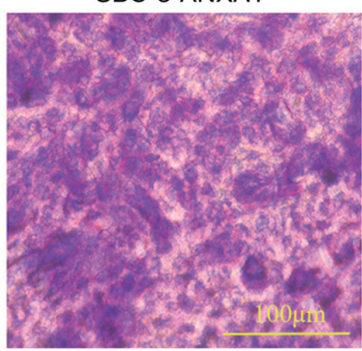

SBC-5-shANXA1

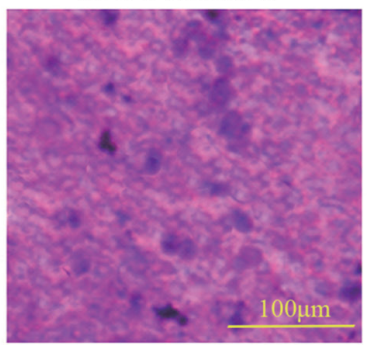

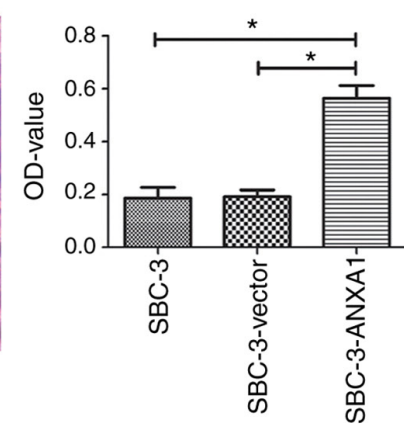

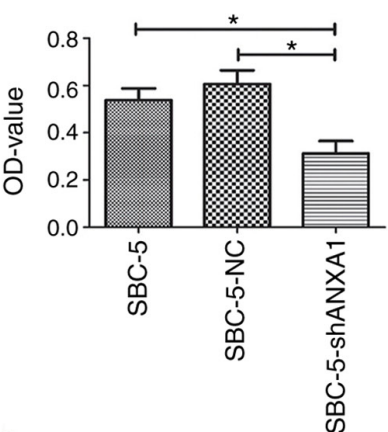

C

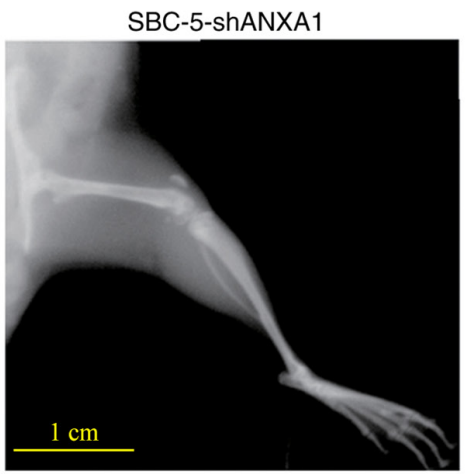

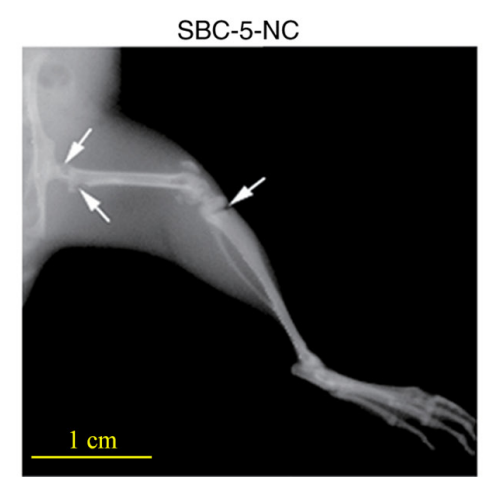

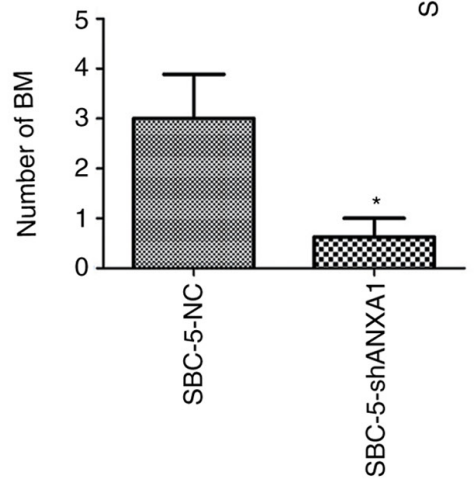

Figure 3. Association between ANXA1 expression and bone metastasis of SCLC. Representative images and quantification of bone adhesion assay in (A) SBC-3 and (B) SBC-5 cells with overexpressed or inhibited ANXA1 expression in vitro, respectively. Scale bar, $100 \mu \mathrm{m}$. (C) Representative images and quantification of xenograft bone metastasis assay in vivo. Scale bar, $1 \mathrm{~cm}$. ${ }^{*} \mathrm{P}<0.05$. ANXA1, annexin A1; SCLC, small cell lung cancer; OD, optical density; NC, negative control; sh, short hairpin; BM, bone metastases.

detected in patients with other organ metastasis. Second, ROC curve analysis revealed that ANXA1 had diagnostic significance for bone metastasis of SCLC. Additionally, in SBC-3/SBC-5 cell lines with similar genetic background and significantly different bone metastasis ability, ANXA1 expression levels were also associated with bone metastasis abilities. Previous studies have confirmed that ANXA1 is a potential tumor biomarker with abnormal expression in a variety of tumors and is associated with multiple clinical factors, such as metastasis and prognosis $(6,11,24)$. However, the expression and function of ANXA1 are not consistent among different tumors. For example, ANXA1 expression is increased in lung adenocarcinoma, gastric cancer and hepatocellular carcinoma, which is associated with a poor prognosis and decreased disease-free survival and metastasis-free survival (25-27). By contrast, several studies reported that ANXA1 expression is downregulated in head and neck squamous cell carcinoma, nasopharyngeal carcinoma and esophageal carcinoma, and is associated with the differentiation grades (28-30). The aforementioned studies suggest that the use of ANXA1 as a therapeutic or prognostic marker for cancer should be carefully evaluated depending on cancer type, grade and stage.

Next, a series of in vivo and in vitro experiments were conducted to further confirm the role of ANXA1 in bone metastasis. By regulating the expression levels of ANXA1 in SCLC cell lines, the present study confirmed that ANXA1 exerted promoting effects on the proliferation, migration and invasion of SCLC cells. Similar results have been reported in previous studies $(9,31)$. For example, autophagy induced by ANXA1 inhibition promoted nasopharyngeal carcinoma cell invasion and metastasis (32). Bone adhesion experiments in vitro and xenograft bone metastasis assay in vivo further confirmed the role of ANXA1 in promoting bone metastasis in SCLC, consistent with the present results of serological testing on patients.

Bone tissue has a distinct structure and composition; hence, the mechanism involved in bone metastasis is different from that of other organs. PTHrP stimulates osteoclastic bone resorption, resulting in bone destruction and TGF- $\beta$ 
A

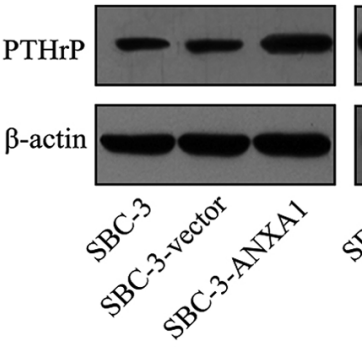

B

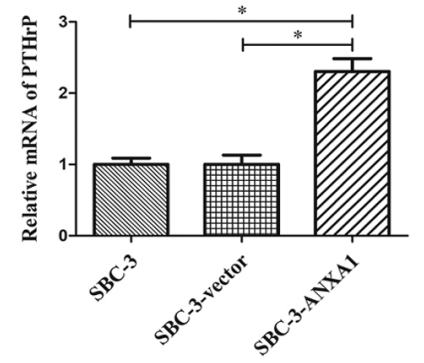

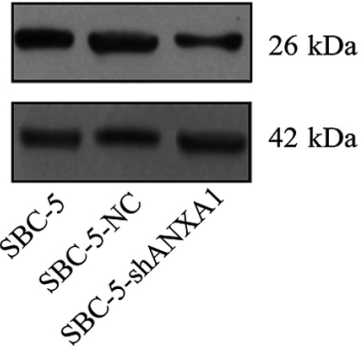

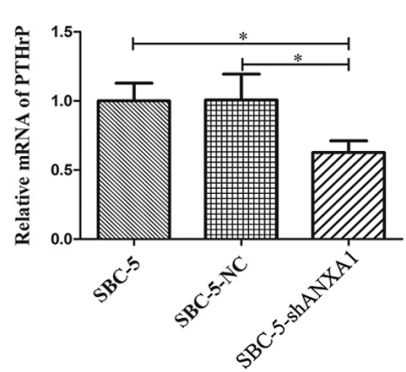

D

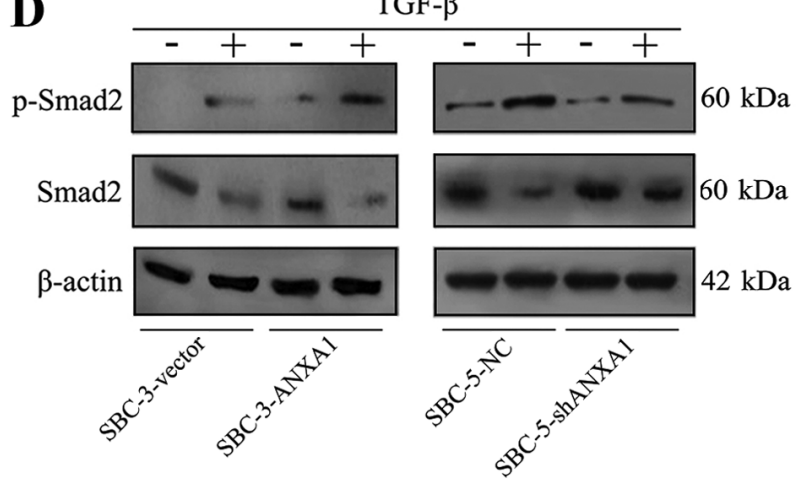

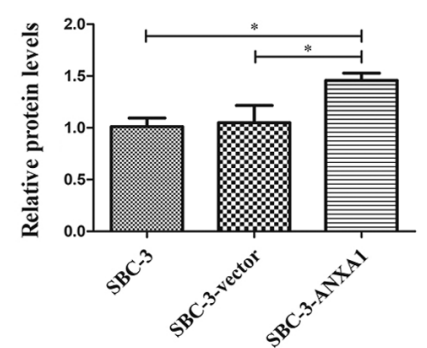

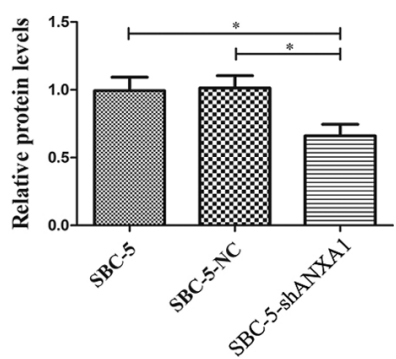

C
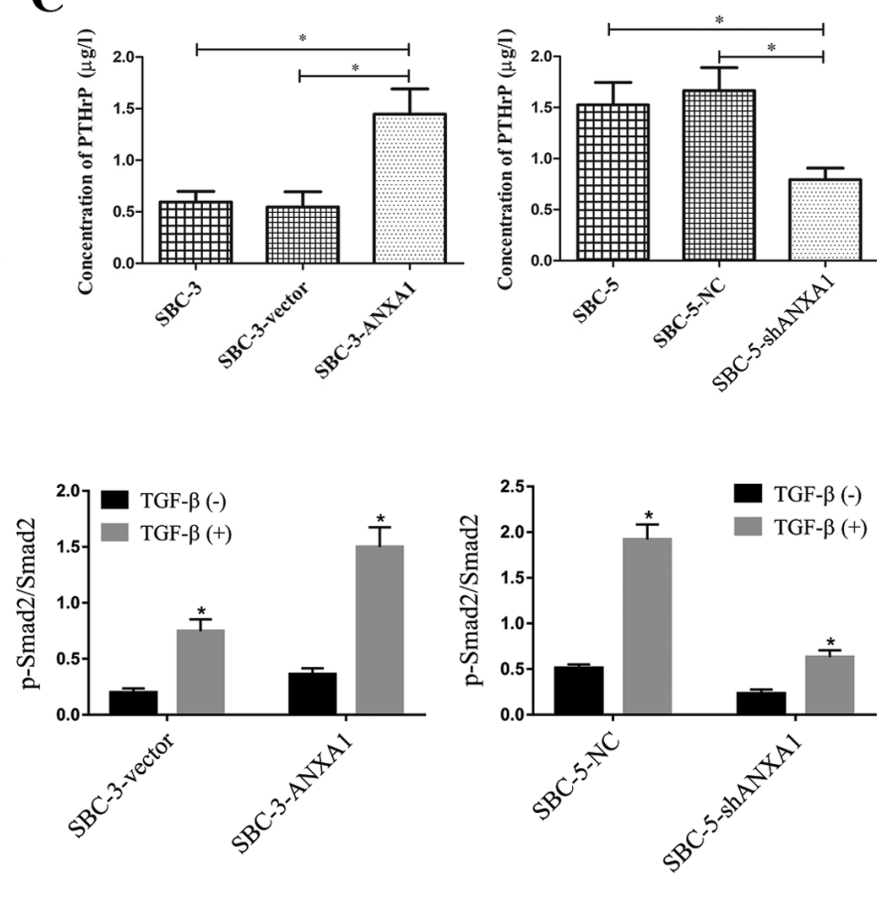

Figure 4. ANXA1-mediated PTHrP and p-Smad2 expression in SCLC cells. (A) Western blotting, (B) reverse transcription-quantitative PCR and (C) ELISA of PTHrP expression in SCLC cells. (D) Western blot analysis of Smad 2 and p-Smad 2 in SCLC cells treated with TGF- $\beta$. ${ }^{*}$ P $<0.05$ vs. TGF- $\beta$ (-). ANXA1, annexin A1; SCLC, small cell lung cancer; PTHrP, parathyroid hormone-related protein; p, phosphorylated; NC, negative control; sh, short hairpin.

release, which is stored in the bone matrix $(33,34)$. TGF- $\beta$ binds to receptors on the surface of tumor cells and induces the phosphorylation of Smad family members (such as the production of $\mathrm{p}$-Smad2), which then activates downstream signaling pathways and regulates the expression of bone metastasis-associated target genes $(21,35)$. The expression levels of PTHrP and p-Smad2 are closely associated with the degree of bone metastasis (21). To confirm the association between ANXA1 and bone metastasis at the mechanistic levels, the effects of ANXA1 on PTHrP and p-Smad2 expression in SCLC cells were assessed. Using western blotting, RT-qPCR and ELISA, the present study confirmed that ANXA1-overexpressing SCLC cells secreted higher levels of PTHrP, while PTHrP synthesis in ANXA1-knockdown SCLC cells was significantly suppressed. In addition, following exposure to TGF- $\beta$, ANXA1-overexpressing SCLC cells exhibited higher levels of p-Smad 2 compared with in ANXA1-knockdown SCLC cells.

The present study has some limitations. First, the current study was a single-center study with a limited clinical sample size. Second, due to the limitations of specimen collection, the present study failed to assess the expression levels of ANXA1,
PTHrP and pSMAD in tumor tissues, nor the association between ANXA1 and other markers, such as PTHrP in serum. Post-mortem studies of tissue samples may be useful to overcome these problems. Third, the expression level of ANXA1 in patients was not continuously monitored, and methods such as Kaplan-Meier analysis were not used to reflect the influence of ANXA1 on the incidence of bone metastasis. Finally, ANXA1 secretion has not been studied thoroughly. Detection of ANXA1 secretion under different conditions using neutralizing antibodies may help to further clarify the function of ANXA1. In addition, the downstream signaling pathways of ANXA1 in promoting bone metastasis, such as the specific role of PTHrP/Smad2, lack of in-depth study. Overcoming these limitations is important to further confirm the role of ANXA1 in bone metastasis and should be further investigated in future studies.

In conclusion, the present study confirmed the strong association between ANXA1 expression and bone metastasis in SCLC from different perspectives, suggesting that ANXA1 may be a potential marker of bone metastasis and providing new potential for the diagnosis and treatment of SCLC. 


\section{Acknowledgements}

Not applicable.

\section{Funding}

The present study was supported by the National Natural Science Foundation of China (grant no. 81702246) and the Key Research and Development Program in Shaanxi Province (grant no. 2019SF-069).

\section{Availability of data and materials}

The datasets used and/or analyzed during the current study are available from the corresponding author on reasonable request.

\section{Authors' contributions}

PC, JM and FZ conceived and designed the experiments. PC, JM, CW, GT and FZ performed the experiments and collected the data. HZ and HW analyzed and interpreted the data. PC, HW and FZ drafted the initial manuscript and revised it for important intellectual content. All authors read and approved the final manuscript.

\section{Ethics approval and consent to participate}

The research involving human samples was approved by the ethical review board of Tangdu Hospital (approval no. TDLL-2017024) and was performed according to the Declaration of Helsinki. All participants signed informed consent. All animal experimental procedures were approved by the Animal Ethics Committee of the Air Force Military Medical University and were in accordance with the 'Animal Research: Reporting In Vivo Experiments' guidelines.

\section{Patient consent for publication}

Not applicable.

\section{Competing interests}

The authors declare that they have no competing interests.

\section{References}

1. Siegel RL, Miller KD and Jemal A: Cancer statistics, 2020. CA Cancer J Clin 70: 7-30, 2020.

2. van Meerbeeck JP, Fennell DA and De Ruysscher DK: Small-cell lung cancer. Lancet 378: 1741-1755, 2011.

3. Wang S, Zimmermann S, Parikh K, Mansfield AS and Adjei AA: Current diagnosis and management of small-cell lung cancer. Mayo Clin Proc 94: 1599-1622, 2019.

4. Conen K, Hagmann R, Hess V, Zippelius A and Rothschild SI: Incidence and predictors of bone metastases (BM) and skeletal-related events (SREs) in small cell lung cancer (SCLC): A swiss patient cohort. J Cancer 7: 2110-2116, 2016.

5. Scagliotti GV, Hirsh V, Siena S, Henry DH, Woll PJ, Manegold C, Solal-Celigny P, Rodriguez G, Krzakowski M, Mehta ND, et al: Overall survival improvement in patients with lung cancer and bone metastases treated with denosumab versus zoledronic acid: Subgroup analysis from a randomized phase 3 study. J Thorac Oncol 7: 1823-1829, 2012.
6. Zhang L and Gong Z: Clinical characteristics and prognostic factors in bone metastases from lung cancer. Med Sci Monit 23: 4087-4094, 2017.

7. Sugimoto MA, Ribeiro ALC, Costa BRC, Vago JP, Lima KM, Carneiro FS, Ortiz MMO, Lima GLN, Carmo AAF, Rocha RM, et al: Plasmin and Plasminogen induce macrophage reprogramming and regulate key steps of inflammation resolution via annexin A1. Blood 129: 2896-2907, 2017.

8. Foo SL, Yap G, Cui J and Lim LHK: Annexin-A1-A blessing or a curse in cancer? Trends Mol Med 25: 315-327, 2019.

9. Guo C, Liu S and Sun MZ: Potential role of Anxal in cancer. Future Oncol 9: 1773-1793, 2013.

10. Mussunoor S and Murray GI: The role of annexins in tumour development and progression. J Pathol 216: 131-140, 2008.

11. Boudhraa Z, Bouchon B, Viallard C, D'Incan M and Degoul F: Annexin A1 localization and its relevance to cancer. Clin Sci (Lond) 130: 205-220, 2016.

12. Damazo AS, Moradi-Bidhendi N, Oliani SM and Flower RJ: Role of annexin 1 gene expression in mouse craniofacial bone development. Birth Defects Res A Clin Mol Teratol 79: 524-532, 2007.

13. Pan X, Liu P and Yin G: Downregulation of annexin A1 by short hairpin RNA inhibits the osteogenic differentiation of rat bone marrow-derived mesenchymal stem cells. Int J Mol Med 36: 406-414, 2015.

14. Detterbeck FC, Boffa DJ, Kim AW and Tanoue LT: The eighth edition lung cancer stage classification. Chest 151: 193-203, 2016.

15. Livak KJ and Schmittgen TD: Analysis of relative gene expression data using real-time quantitative PCR and the 2(-Delta Delta C(T)). method. Methods 25: 402-408, 2001.

16. Ma N, Shen W, Pang H, Zhang N, Shi H, Wang J and Zhang H: The effect of RCAN1 on the biological behaviors of small cell lung cancer. Tumour Biol 39: 1010428317700405, 2017.

17. Kilkenny C, Browne W, Cuthill IC, Emerson M and Altman DG; NC3Rs Reporting Guidelines Working Group: Animal research: Reporting in vivo experiments: The ARRIVE guidelines. Br J Pharmacol 160: 1577-1579, 2010.

18. Miki T, Yano S, Hanibuchi M and Sone S: Bone metastasis model with multiorgan dissemination of human small-cell lung cancer (SBC-5) cells in natural killer cell-depleted SCID mice. Oncol Res 12: 209-217, 2000

19. Mak IW, Turcotte RE and Ghert M: Parathyroid hormone-related protein (PTHrP) modulates adhesion, migration and invasion in bone tumor cells. Bone 55: 198-207, 2013.

20. Frieling JS, Shay G, Izumi V, Aherne ST, Saul RG, Budzevich M, Koomen J and Lynch CC: Matrix metalloproteinase processing of PTHrP yields a selective regulator of osteogenesis, $\mathrm{PTHrP}_{1-17}$ Oncogene 36: 4498-4507, 2017.

21. Kakonen SM, Selander KS, Chirgwin JM, Yin JJ, Burns S, Rankin WA, Grubbs BG, Dallas M, Cui Y and Guise TA: Transforming growth factor-beta stimulates parathyroid hormone-related protein and osteolytic metastases via Smad and mitogen-activated protein kinase signaling pathways. J Biol Chem 277: 24571-24578, 2002.

22. Zhao X, Kallakury B, Chahine JJ, Hartmann D, Zhang Y, Chen Y, Zhang H, Zhang B, Wang C and Giaccone G: Surgical resection of SCLC: Prognostic factors and the tumor microenvironment. J Thorac Oncol 14: 914-923, 2019.

23. Katakami N, Kunikane H, Takeda K, Takayama K, Sawa T, Saito H, Harada M, Yokota S, Ando K, Saito Y, et al: Prospective study on the incidence of bone metastasis (BM) and skeletal-related events (SREs) in patients (pts) with stage IIIB and IV lung cancer-CSP-HOR 13. J Thorac Oncol 9: 231-238, 2014.

24. Sobral-Leite M, Wesseling J, Smit VT, Nevanlinna H, van Miltenburg MH, Sanders J, Hofland I, Blows FM, Coulson P, Patrycja G, et al: Annexin A1 expression in a pooled breast cancer series: Association with tumor subtypes and prognosis. BMC Med 13: 156, 2015.

25. Biaoxue R, Xiling J, Shuanying Y, Wei Z, Xiguang C, Jinsui W and Min Z: Upregulation of Hsp90-beta and annexin A1 correlates with poor survival and lymphatic metastasis in lung cancer patients. J Exp Clin Cancer Res 31: 70, 2012.

26. Lin $\mathrm{Y}$, Lin $\mathrm{G}$, Fang $\mathrm{W}$, Zhu $\mathrm{H}$ and Chu $\mathrm{K}$ : Increased expression of annexin A1 predicts poor prognosis in human hepatocellular carcinoma and enhances cell malignant phenotype. Med Oncol 31: 327, 2014.

27. Sato Y, Kumamoto K, Saito K, Okayama H, Hayase S, Kofunato Y, Miyamoto K, Nakamura I, Ohki S, Koyama Y and Takenoshita S: Up-regulated annexin A1 expression in gastrointestinal cancer is associated with cancer invasion and lymph node metastasis. Exp Ther Med 2: 239-243, 2011. 
28. GarciaPedrero JM,Fernandez MP, Morgan RO,Herrero Zapatero A Gonzalez MV, Suarez Nieto C and Rodrigo JP: Annexin A1 down-regulation in head and neck cancer is associated with epithelial differentiation status. Am J Pathol 164: 73-79, 2004.

29. Rodrigo JP, Garcia-Pedrero JM, Fernandez MP, Morgan RO, Suarez C and Herrero A: Annexin A1 expression in nasopharyngeal carcinoma correlates with squamous differentiation. Am J Rhinol 19: 483-487, 2005.

30. Xia SH, Hu LP, Hu H, Ying WT, Xu X, Cai Y, Han YL, Chen BS, Wei F, Qian XH, et al: Three isoforms of annexin I are preferentially expressed in normal esophageal epithelia but down-regulated in esophageal squamous cell carcinomas. Oncogene 21: 6641-6648, 2002.

31. Bizzarro V, Belvedere R, Migliaro V, Romano E, Parente L and Petrella A: Hypoxia regulates ANXA1 expression to support prostate cancer cell invasion and aggressiveness. Cell Adh Migr 11: 247-260, 2017.

32. Zhu JF, Huang W, Yi HM, Xiao T, Li JY, Feng J, Yi H, Lu SS, Li XH, Lu RH, et al: Annexin A1-suppressed autophagy promotes nasopharyngeal carcinoma cell invasion and metastasis by PI3K/AKT signaling activation. Cell Death Dis 9: 1154, 2018.
33. Guise TA, Mohammad KS, Clines G, Stebbins EG, Wong DH, Higgins LS, Vessella R, Corey E, Padalecki S, Suva L and Chirgwin JM: Basic mechanisms responsible for osteolytic and osteoblastic bone metastases. Clin Cancer Res 12: 6213s-6216s, 2016.

34. Roodman GD: Biology of osteoclast activation in cancer. J Clin Oncol 19: 3562-3571, 2001.

35. Kunihiro AG, Brickey JA, Frye JB, Luis PB, Schneider C and Funk JL: Curcumin, but not curcumin-glucuronide, inhibits Smad signaling in TGF $\beta$-dependent bone metastatic breast cancer cells and is enriched in bone compared to other tissues. J Nutr Biochem 63: 150-156, 2019.

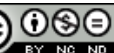

This work is licensed under a Creative Commons Attribution-NonCommercial-NoDerivatives 4.0 International (CC BY-NC-ND 4.0) License. 